\title{
Cloning and characterization of a novel sperm tail protein, NYD-SP28
}

\author{
YING ZHENG $^{1,2}$, JINGJING ZHANG $^{1}$, LEI WANG ${ }^{1}$, ZUOMIN ZHOU $^{1}$, MIN XU $^{1}$, \\ JIANMIN LI ${ }^{1}$ and JIA-HAO SHA ${ }^{1}$ \\ ${ }^{1}$ Laboratory of Reproductive Medicine, Department of Histology and Embryology, \\ Nanjing Medical University, Nanjing 210029, ${ }^{2}$ Department of Histology and Embryology, \\ School of Medicine, Yangzhou University, Yangzhou 225001, P.R. China
}

Received June 1, 2006; Accepted July 27, 2006

\begin{abstract}
In this study, a gene coding a novel human sperm tail protein named NYD-SP28 was cloned and characterized using a complementary DNA (cDNA) microarray. Its expression was 3.5 times higher in human testis than in fetal testis, and very high in human spermatozoa. The full length of NYD-SP28 cDNA was 1798 bp and encoded a 484amino-acid protein. Motif analysis revealed that the protein contained a cluster of phosphorylation sites, N-glycosylation sites and $\mathrm{N}$-myristoylation sites. Immunohistochemical analysis of normal human testes showed that NYD-SP28 was expressed in the cytoplasm of spermatogenic cells but not in interstitial cells. The EGFP-NYD-SP28 fusion protein was also localized in the cytoplasm of transfected 7721 cells. In human spermatozoa, NYD-SP28 immunoreactivity was detected in entire sperm tail. Using the two-dimensional (2-D) gel electrophoresis and immunoblotting technique, NYDSP28 was found to be post-translationally modified during sperm capacitation. In conclusion, these results suggest that NYD-SP28 is a new human sperm tail protein and might play an important role during sperm capacitation.
\end{abstract}

\section{Introduction}

Ejaculated sperm must spend additional time in the female tract, or another appropriate environment, before they are competent to fertilize. This final maturation process was termed capacitation (1). During this process, extensive changes occurred in all sperm compartments: a) factors originating from epididymal fluid and seminal plasma were lost or redistributed, b) membrane lipids and proteins were reorganized, c) ion fluxes induced biochemical modifications,

Correspondence to: Dr Jianmin Li, Laboratory of Reproductive Medicine, Nanjing Medical University, Nanjing 210029, P.R. China

E-mail: jianminli@njmu.edu.cn

Key words: NYD-SP28, testis, expression, sperm capacitation and controlled amounts of reactive oxygen species were generated, d) complex signal transduction mechanisms were initiated, and e) spermatozoa developed hyperactivated motility and the acrosome reaction (2). An intriguing facet of this acquisition of functional competence was that it occurred in the complete absence of contemporaneous gene transcription and translation in spermatozoa. Hence, it is widely accepted that post-translational modifications are the only means by which spermatozoa can acquire functionality (3). In the human, various proteins have been cloned and found to be modified during capacitation, among which fibrous sheath proteins P81, P95 and p105 are most abundant. However, mechanisms that control sperm capacitation are not yet completely known (2).

In our previous studies, we compared gene expression profiles between adult and fetal testes by hybridizing cDNA probes (prepared from adult and fetal testes) to membranes fabricated with gene clones from the human testis library (4-7). In the present study, we report a novel human gene, named NYD-SP28, which is expressed in human sperm tail and may play an important role during sperm capacitation. The genomic mapping, computer-assisted analyses of the protein structure, its distribution in the cell and adult tissue, human testis and spermatozoa were assessed. By the combination of two-dimensional gel electrophoresis and immunoblotting, protein expression in noncapacitated and capacitated human spermatozoa was also examined.

\section{Materials and methods}

Samples. All samples were collected after ethical approval was granted by the Committee of Nanjing Medical University on Human Rights in Research, and consent forms were signed by all participants.

Human adult testes were obtained from the Body Donor Center (Nanjing Medical University, Nanjing, P.R. China), and fetal testes were obtained from accidentally aborted six-monthold fetuses (Clinical Reproductive Center, Nanjing Medical University). Macroscopic and histological examinations were performed to confirm the structural integrity of these samples.

Human normal ejaculates were obtained from healthy volunteers with proven fertility and normal semen quality based on WHO criteria (1999), which is $>20 \times 10^{6}$ spermatozoa/ 
$\mathrm{ml}$ with $>50 \%$ active sperm, $>25 \%$ sperm moving forcefully in one direction (rapid and linearly progressive), $>30 \%$ sperm with normal morphology and $<1$ lymphocyte per high power field (40x).

Preparation of human testis cDNA microarray. A total of 9,216 positive phage clones were chosen randomly from human testis large insert $\lambda$ phage cDNA library (Clontech, HL5503U) and then converted into pTriplEx 2 clones (according to Clontech's manual PT3003-1). The inserts were amplified by polymerase chain reaction (PCR) and then used to make human testis cDNA microarray. Protocols for human testis cDNA microarray construction, human embryo and adult testis cDNA probe preparation, hybridization, and signal analysis have been well described in our previous study (4).

Sequencing and analysis of clones. The differentially expressed cDNA plasmid was extracted and purified via mini-prep kits (QIAprep Spin miniprep kit; Qiagen). The full length of the insert was sequenced by an autosequencer (ABI model 377, Perkin-Elmer, Norwalk, CT). The generated sequences were subjected to BLAST analysis (http://www. ncbi.nlm.nih.gov) to determine the sequence homology with other species and the gene locus on the human chromosome. The nucleotides and deduced protein were analyzed by Gene Runner (http://www. generunner.com). After sequence identification and analysis, a novel gene, named NYD-SP28, was found. Its complete sequence has been accepted by GenBank with the accession number AF382188.

Construction and transfection of pEGFP-C3-NYD-SP28 to 7721 cells. The NYD-SP28 open reading fragment was cloned into the XhoI-SalI sites of pGEFP-C3 vector (Clontech Laboratories, Palo Alto, CA). The constructed EGFP-NYDSP28 cDNA was transfected into 7721 cells using a Qiagen transfection kit as described previously (5). As a control, pEGFP-C3 vector without insert was transfected into cells in separate wells simultaneously under the same condition. GFP fusion protein was visualized under a Laser confocal microscope (Zeiss, Gottingen, Germany).

Production of recombinant NYD-SP28 protein. The open reading frame encoding human NYD-SP28 was cloned into the BamHI-EcoRI sites of the pET28a expression vector (Novagen, Madison, WI) and used to transform Escherichia coli BL21 (DE3). Recombinant protein expression was induced for $3 \mathrm{~h}$ with $1.0 \mathrm{mM}$ isopropyl-1-thio-b-D-galactopyranoside. The cells were then transferred to PBS and briefly treated by sonicate. The inclusion bodies collected by centrifugation at $15,000 \mathrm{x}$ g for $15 \mathrm{~min}$ were dissolved in solubilization buffer ( $8 \mathrm{M}$ urea, $20 \mathrm{mM}$ Tris- $\mathrm{HCl}, \mathrm{pH} 7.9$, $0.5 \mathrm{M} \mathrm{NaCl}$ ) for $1 \mathrm{~h}$ on ice and followed by sonicate. After centrifugation at $15,000 \mathrm{x} \mathrm{g}$ for $15 \mathrm{~min}$, the urea soluble fraction was adsorbed to the Ni21-activated His-binding resin (Shengbo Co., Shanghai, P.R. China) which was then eluted with $200 \mathrm{mM}$ imidazole in solubilization buffer containing $8 \mathrm{M}$ urea. Finally, the purified recombinant protein was refolded by gradually decreased urea series (from $7 \mathrm{M}$ to $2 \mathrm{M}$ ).

Purified recombinant NYD-SP28-His $(\sim 100 \mu \mathrm{g})$ was used to immunize mice. The mice were sacrificed 10 days after the last boost, and titers (by ELISA) above 1:10,000 were observed in blood collected from all immunized mice. Serum from one mouse was used for antibody purification via an Affi-gel Protein A MAPS II kit (BioRad, Hercules, CA) and subsequently used for immunohistochemical analysis.

Immunohistochemistry. Immunohistochemical localization of NYD-SP28 protein was assessed using a Vectastain Elite $\mathrm{ABC}$ kit according to the manufacturer's instructions. Briefly, the testis was fixed in $10 \%$ formaldehyde and embedded in paraffin. Sections (3-4 $\mu \mathrm{m}$ in thickness) were deparaffinized in xylene, rehydrated in graded ethanol series, and rinsed in tap water. Endogenous peroxidase activity was blocked using $3 \% \mathrm{H}_{2} \mathrm{O}_{2}$. After rinsing in tap water, the sections were microwaved for $2 \mathrm{~min}$ in $10 \mathrm{mM}$ citrate buffer ( $\mathrm{pH} 6.0$ ), cooled for $20 \mathrm{~min}$, and again rinsed in tap water for antigen retrieval. Sections were then blocked with goat serum (Beijing ZhongShan Biotechnology Co. Ltd., Beijing, P.R. China) for $1 \mathrm{~h}$ at RT and incubated with primary antibody (antihuman polyclonal NYD-SP28, 1:1000 dilution) overnight at $4^{\circ} \mathrm{C}$. The sections were further incubated with HRP-conjugated goat anti-mouse secondary antibody (1:2000 dilution, Beijing ZhongShan Biotechnology) for $40 \mathrm{~min}$ at room temperature. Sections serving as negative controls were incubated with dilution buffer in the absence of primary antibody. Between each step, sections were washed in PBS for three times at RT. Finally, counterstaining was carried out with hematoxylin, and coverslips were applied using aqueous mounting media.

Indirect immunofluorescence. Spermatozoa were washed three times with PBS by centrifugation ( $500 \mathrm{x} \mathrm{g,} 10 \mathrm{~min}$ ), airdried on microscope slides, and fixed for $30 \mathrm{~min}$ in PBS with $4 \%$ paraformaldehyde. Cells were then permeated for $20 \mathrm{~min}$ in PBS containing $0.2 \%$ Triton X-100. After rinsing with PBS, slides were placed in blocking buffer (goat serum, Beijing ZhongShan Biotechnology) for $2 \mathrm{~h}$ at room temperature and then incubated with anti-NYD-SP28 polyclonal antibodies (diluted 1:1000 in blocking buffer) overnight at $4^{\circ} \mathrm{C}$. After three washes in PBS containing $0.05 \%$ Tween-20 (PBST), slides were incubated with secondary goat anti-mouse antibody labeled with a fluorescent dye (Molecular Probes, Eugene, OR) for $40 \mathrm{~min}$ at room temperature. The slides were then washed, mounted with distilled water, and examined under a Zeiss Axiovert 100-M inverted microscope equipped with a LSM510 laser unit.

Western blot analysis. Frozen human testis tissues were homogenized in lysis buffer $(7 \mathrm{~mol} / 1$ urea, $2 \mathrm{~mol} / 1$ thiourea, 4\% (v/v) CHAPS, 65 mmol/1 DTT), and multiple protease cocktail inhibitors (PIERCE) were added at the same time. After homogenization the samples were centrifuged at 20,000 x g for $10 \mathrm{~min}$ at $4^{\circ} \mathrm{C}$. The supernatants were designated as soluble protein extracts. The protein concentration was determined using the method of Bradford.

Protein samples were diluted in SDS-PAGE buffer and boiled for $5 \mathrm{~min}$ before being loaded (40 $\mu \mathrm{g} / \mathrm{lane}$ ) onto the gels $(12.5 \%)$. Proteins were then transferred electrophoretically by semidry blot to nitrocellulose membranes. After incubation with a blocking buffer (phosphate-buffered 


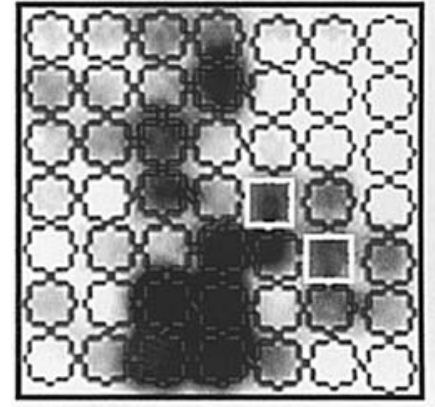

A

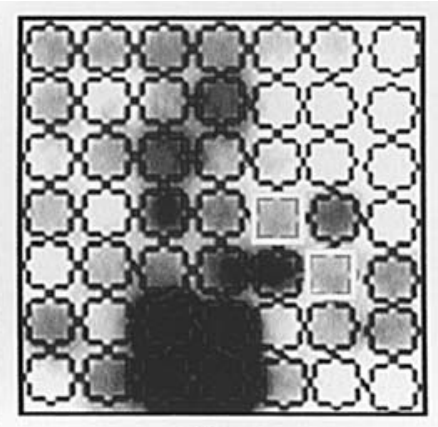

B
Figure 1. cDNA hybridization images showing differential expression of NYD-SP28 in adult human testes (A) and 6-month-old fetal testes (B). The white panels indicate that the NYD-SP28 cDNA and the hybridization intensities in adult and fetal testes are 76.9 and 22.11 , respectively. Hybridization intensity was 3.5 -fold higher in adult than in fetal testes.

saline containing $5 \%$ non-fat milk), the nitrocellulose membranes were probed overnight at $4^{\circ} \mathrm{C}$ with NYD-SP28 polyclonal antiserum. After extensive washing, the membranes were incubated with HRP-conjugated secondary antibody (Bio-Rad, CA, USA) for $1 \mathrm{~h}$ at room temperature in $1 \%$ non-fat milk. The signals were visualized by the enhanced chemiluminescence system (ECL, Amersham, Uppsala, Sweden).

Preparation of spermatozoa. Human semen samples were collected from healthy fertile donors after 3 days of sexual abstinence. After semen liquefaction ( $\sim 30 \mathrm{~min})$, the sperm ejaculates were loaded onto a 40-85\% Percoll gradient in modified BWW medium (Quinn et al, 1985) with $10 \%$ fetal bovine serum (Fraction V; Sigma Chemical Co., St. Louis, MO). The samples were washed through the gradient at $500 \mathrm{x} \mathrm{g}$ for $18 \mathrm{~min}$, collected from both the pellet and the $85 \%$ Percoll layer, and then washed again by centrifugation with BWW to remove the Percoll at $800 \mathrm{x}$ g for $5 \mathrm{~min}$. The prepared samples were divided into two parts. One part was immediately fixed in $4 \%$ paraformaldehyde or solubilized directly in lysis buffer and the resultant supernatant was designated as noncapacitated sperm proteins. The other part was capacitated for $5 \mathrm{~h}$ in $\mathrm{BWW}$ media at $37^{\circ} \mathrm{C}$ in a $5 \% \mathrm{CO}_{2}$ atmosphere. Then the incubated sperm (capacitated) were fixed in $4 \%$ paraformaldehyde or solubilized directly in lysis buffer.

Two-dimensional gel electrophoresis. Protein extraction and immobilized $\mathrm{pH}$ gradient two-dimensional gel electrophoresis were performed as described previously (8). Proteins (two repetitions of each) were then transferred electrophoretically by semidry blot to nitrocellulose membranes, and used for Western blot analysis.

\section{Results}

cDNA microarray hybridization. After hybridization and data analysis, genes differentially expressed in both human adult and fetal testes were considered as testis development and/or spermatogenesis related. A clone, named NYD-SP28, was identified. Its expression was high in adult testis but low in
GGGGGGGAGAGCTCAGCCTCACTGATAGCCGGGGATCTCTCCTGTTCGAGGGCTGAGATC TTCGGTCCCACAACCGCCCACAACCAGGGGCACTTCTGGAAGTCCCCTTTCTAGGGACAT TTTTCTTCTAAGCTCTGTAACGCGGGCCGAGGCAGACCGAGGCTTCTTTCCCGGATCCAA TCCCCTGCCCCCATGCCTAAGAAAGAAAAAATGGCCAAGACGCCCCTGTCCGATGAGAAG M $P$ P CAGCTGCTTCTGTTTCAGCAGAAGTTGCTGGCAGAGGAGGAGATGGCCAAAAAGAAGGAG

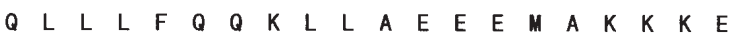
AGGCTCCTCAGCCAGTTCTTGAAGGAGAAGCTGGCCAAGGAGGAACACAACAGTGCTCTG R L L S S Q AACCTTAATAAGATTAACACACAGTGGAGAACTGTCCTTCGgGAAGTCAAGACCAGAGAA $\begin{array}{llllllllllllllllllll}N & L & N & K & \text { I } & N & T & Q & \text { W } & R & T & V & L & R & E & V & K & T & R & E\end{array}$ CTTCATAAGGACATTGAGATCCTCAGCCAAACATTTGAAGGAGTGGTGGACTGTAAGGAC L $H$ K K D I $\quad E$ I $L$ AATGTCATCAAGTCTTTAGCTAAAGACCTGTCCGAAGCCGAGGAGCAGTACGCCCATGCC N $V$ I $K$ K $S$ S L A CTGCGCAGCCACTTGCACAATGTTGACCAGCTCTTGGCCCTGCAGAGGCACCGGCTCAGT

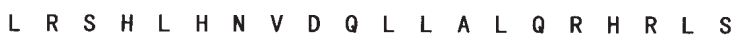
CTCCTGGAGGAAAGTTACAACATGGAGGTGGAAGCCCTAACCAAGGAGTTTGAGACAGAA $L \quad L \quad E$ E $S$ Y Y N $N$ M $E$ L $L$ E A AGgAAGACAATTATTGACCAACATGAGAAAGAGATTCACTATCTGCAAGATATCTTCATG $\begin{array}{llllllllllllllllllll}R & K & T & \text { I } & \text { I } & D & Q & \text { H } & E & K & E & \text { I } & \text { H } & \text { Y } & L & Q & \text { Q } & \text { I } & F & M\end{array}$ GGCATGGAGCAGAACTATATAGATTCTGAGTATGAAAGCAAGCTGGAGTTCCAGAGCATG

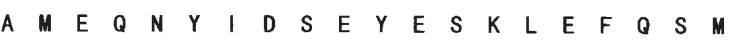
TGGAATGATCTCAAAGACATGAATTTAGAGGAGAGCACTTTCTAAGACTGCACCTGGAG

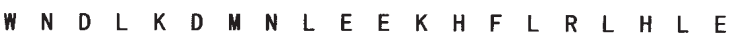
AACAGAGTAGAAGATCTGTGGAGAAAGTTCCAGGATGTACTCAAGAATTACACTGATGCC

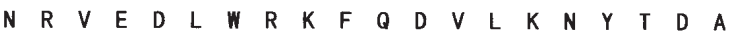
ACAGAGGATCGAAAGACTGCCTTTGAGACCCTGCAGGTGAAGGATGAGAAGAGCTCCAAA

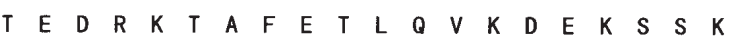
GAGATTGAAGTACAGATGAAAAAAATAGAGAAACTACAGGATGCCATAACTATTTCAAAA E I $E$ E V GGCAAGATCATGATACACAGCCGTGAGAGTGAAGATGAGAACCGGTATATCCGTAATGAC

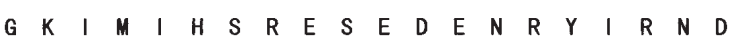
AAGGAATTGGTCCTTGTACAACTGCGAAAACTTAAGGCCCAAAGAACTCAGGCCCGGGCA

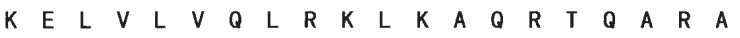
GCATCCCAGAAGAACTTAGTCAGACTCACCCTGGAAAGTAATGCCACCCTCAAGGCCCTG

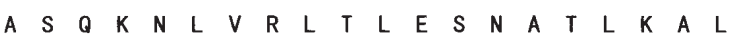
AGAAAGATTGTTGATAAGGGTGAAAAGATCCTTAAAGTTGCTGAAATATGTAGGAAATTT

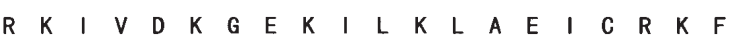
GAAACCGAAGAAGAAAAAGTGCTGCCTTTTTATTCATCAGTATTGACTCCTAAGGAGCAG

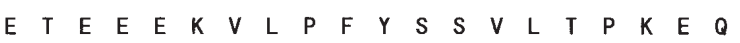
GAGGGGATTCAGAAGAATAATCTAGAAGAGCTTACTGAGGAGCTCACCAAGGTGATGGTG

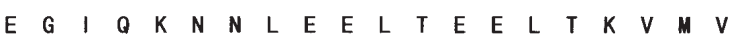
GACTACATAGGAATGGAGAATTTCTGGAAAAGGTACAACAAAGTGAAACTGGAGCAACTG

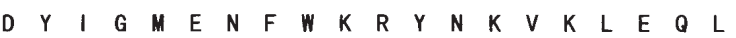
AGCCTCCAACATAGACGAGCCCAGCTGCTAGATATCAATGGGAAGCTGCGGGAGATGCTG

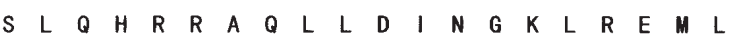
AAGCAGTACTTGGATGGCATCTCAGTGAGTGACGAAGTGCTGAGCCAGCTCAACCCACTC

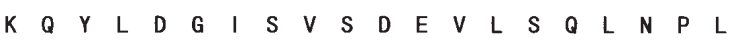
TTTATAGTCAACTATCAAAGCAACTTACTCCAGCCCTTGTCCATACGTATAGCCCATCCA

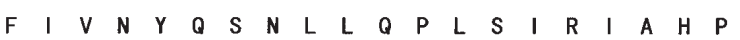
GGTGATAAACAACATCCAACCACTTAAAATATAATTGAAGCAGCCCAGATGATCTTCCAC G $\quad D \quad K \quad$ Q AACCTGTGATCTAAGGAAAAAAATCTITCAACTCCTAGAGATTTTTTTTITTTTTTTGAGA AATGTAGGGATGTTGCTATTAAAAATTTCCATGGAGTTTGgGAAAAAAAAAAAAAAAA

60 120 180 240

300 360 420 480 540 600 660 720 780 840 900 960 1020 1080 1140 1200 1260 1320 1380 1440 1500 1560 1620 1680

Figure 2. Nucleic acid and deduced amino acid sequences of NYD-SP28 cDNA. The number of nucleotides and amino acids are shown on each line. The complete sequence of NYD-SP28 has been published in GenBank with the Accession no. AF382188. 


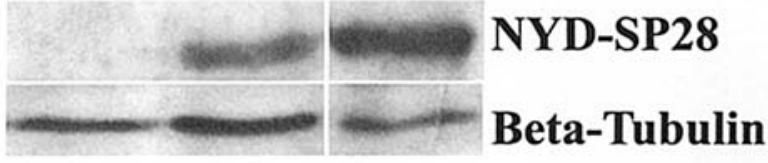

\section{Fetal Adult Sperm}

Figure 3. Immunoblotting analysis of NYD-SP28 protein in human fetal and adult testes. B-tubulin was used as a control. NYD-SP28 protein contents were high in human adult testes but not detectable in human fetal testes. NYD-SP28 specific fragment was also observed in human spermatozoa.

fetal testes (Fig. 1). The signal intensities hybridized with embryo testis probe and with adult testis probe were 22.11 and 76.9, respectively. As shown in Fig. 1, the gene expression was $\sim 3.4$ fold higher in adult testis than in fetal testis.

Sequence identification and analysis of the NYD-SP28 gene. The full nucleotide and amino acid sequences of NYD-SP28 are shown in Fig. 2. NYD-SP28 cDNA consisted of 1798 nucleotides spanning eight exons, and contained an ORF of $1455 \mathrm{bp}$. The ORF encoded a protein of 484 amino acids with an expected molecular weight of $57 \mathrm{kDa}$ and a deduced pI of 7.05. The nucleotide sequence has been published in GeneBank with the accession number AF382188. BLAST search in the human genome database localized NYD-SP28 to human chromosome 12 (NT_029419.10lHs12_29578). It was spliced by 8 exons and 7 introns, encompassing 17427 bp genomic DNA in NT_029419.10. BLAST search of the contig map showed that all exons were located within chromosome $12 \mathrm{q} 13.12$, so this gene was mapped to chromosome 12q13.12. Computer-assisted sequence analysis by GenRunner (version 3.00) showed that NYD-SP28encoded protein contained two glycosylation sites (232th, 330th), two myristoylation sites (378th, 442th), and a cluster of phosphorylation sites, including six protein kinase $\mathrm{C}$ (PKC) phosphorylation sites (155th, 254th, 318th, 332th, 372th, 470th), 11 casein kinase II phosphorylation sites (105th, 136th, 159th, 185th, 189th, 242th, 254th, 286th, 358th, 372th, 444th) and one tyrosine kinase phosphorylation site (284th).

Multiple sequence comparison among different species by program ClustalW and BLASTp revealed that the amino acid sequence of NYD-SP28 proteins in the human, mouse, rat, macaque, cow and dog have a high level of conservation with particular significance in its $\mathrm{N}$ terminus. The potential function of these homologous genes, however, has not been elucidated. Among them mouse NYD-SP28 (AK454432) was also cloned in our laboratory. NYD-SP28 and AK454432 were $81 \%$ and $79 \%$ identical in nucleotide and amino acid sequences, respectively (data not shown). These data indicated that the NYD-SP28 gene was highly conserved during evolution and homologous between the human and the mouse.

Expression profile of NYD-SP28 during testis development. Antibodies were generated against recombinant NYD-SP28 and produced in E. coli. The cross-reactivity and specificity of the antibody were examined by Western blot analysis for endogenous testicular proteins (approximately $57 \mathrm{kDa}$ ) which were the products of cloned NYD-SP28 cDNA.

NYD-SP28 protein content in developing testis was assessed by Western blotting. Results indicated that NYDSP28 levels were high in human adult testes, but not detectable in human fetal testes (Fig. 3). In addition, the

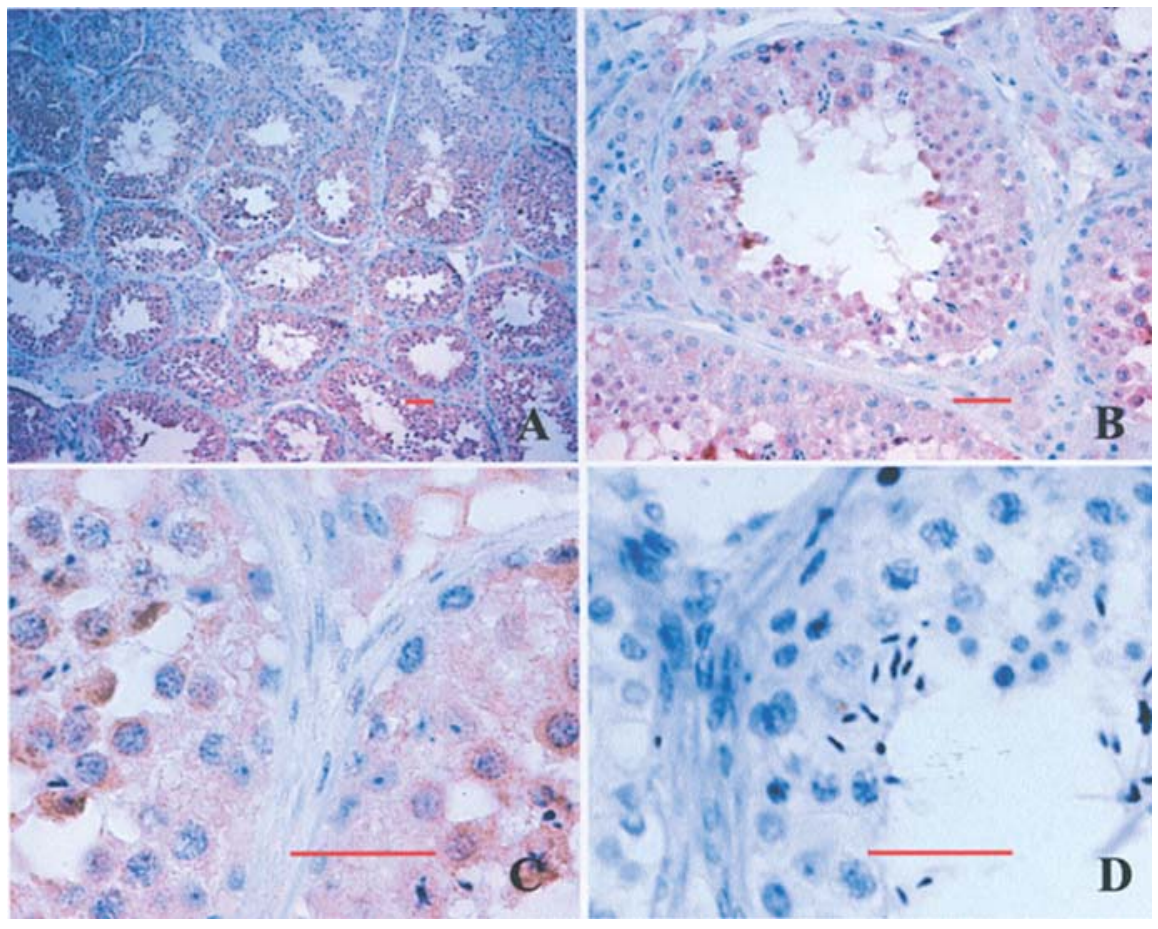

Figure 4. Localization of NYD-SP28 in human testes by immunohistochemical analysis. Sections of human testes were incubated with anti-NYD-SP28 antibody. (A-C) Anti-NYD-SP28 antibody, (D) control (preimmune rabbit serum). Bars $=25 \mu \mathrm{m}$. 


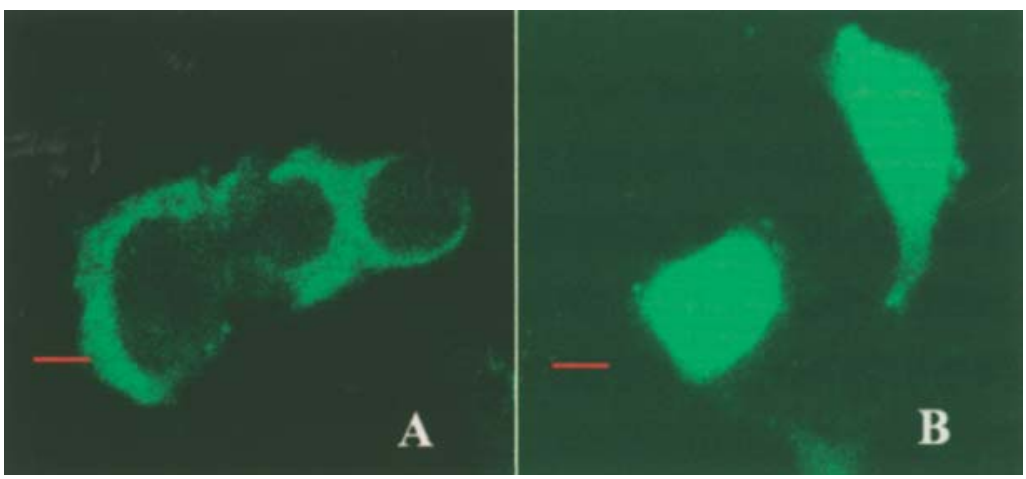

Figure 5. Confocal laser microscopy detection of pEGFP-C3-NYD-SP28 fusion protein transiently expressed in 7721 cells. NYD-SP28 fusion protein was located in the cytoplasm. (A) Plasmid pEGFP-C3-NYD-SP28 recombination vector, (B) plasmid pEGFP-C3 vector (control). Images were visualized under 488-nm fluorescence. Bars $=5 \mu \mathrm{m}$.
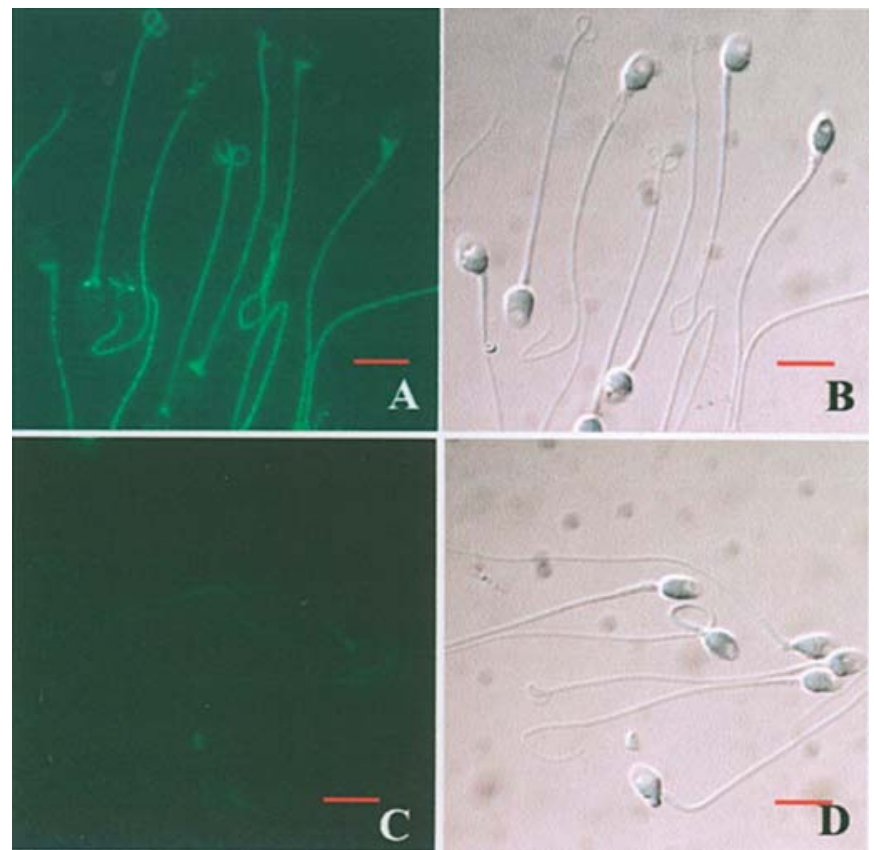

Figure 6. Confocal laser scanning photomicrographs showing the localization of NYD-SP28 in human sperm cells. Anti-NYD-SP28 immunoreactivity by FITC staining was observed in the sperm tail (A and B), but not in negative control (C and D). Bars $=20 \mu \mathrm{m}$.

NYD-SP28 specific fragment was also observed in human spermatozoa.

Immunohistochemistry revealed cellular localization of NYD$S P 28$ protein in human testis. To determine cell types in the testis in which NYD-SP28 protein was expressed, immunohistochemical analysis was carried out using sections of human testis. NYD-SP28 protein was primarily detected in the spermatocyte and spermatid. A weak signal was observed in spermatogonia, and no signal was found in Leydig cells (Fig. 4). Non-specific immunostaining was not observed with the preimmune rabbit antisera (Fig. 4D).

Subcellular localization of NYD-SP28. The subcellular localization of the fusion protein was examined by transient transfection in human 7721 cells. The results demonstrated

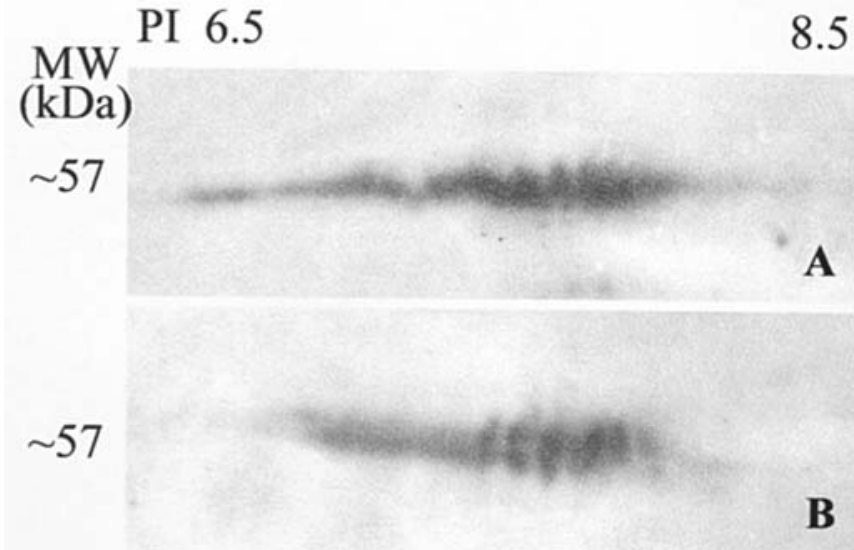

Figure 7. Identification of posttranslationally modified NYD-SP28 protein in noncapacitated (A), and capacitated (B) human spermatozoa by 2-D immunoblotting.

that pEGFP-NYD-SP28 fusion protein was expressed merely in the cytoplasm of the transfected 7721 cell $(24 \mathrm{~h})$. In contrast, the pEGFP-C3 protein (control) was distributed throughout the whole cell without any compartmentalization (Fig. 5).

Localization of NYD-SP28 in human spermatozoa. Indirect immunofluorescence analysis of fresh noncapacitated human spermatozoa showed the existence of NYD-SP28 throughout the entire sperm tail (Fig. 6A). In capacitated and acrosomereacted sperm preparations, the staining patterns of NYDSP28 were the same as that in noncapacitated spermatozoa (data not shown). A non-specific immunofluorescence signal was not detected with the preimmune antisera (Fig. 6C).

NYD-SP28 is post-translationally modified during spermatozoa capacitation. Computer-assisted sequence analysis showed that NYD-SP28-encoded protein contained two glycosylation sites, two myristoylation sites and a cluster of phosphorylation sites. To determine the possible involvement and the nature of posttranslational modifications, further studies were carried out as follows. Proteins extracted from noncapacitated and capacitated human sperm were resolved by 2-D SDSPAGE, electroblotted, and incubated with antisera against 
NYD-SP28, anti-phosphotyrosine, anti-phosphoserine and anti-phosphothreonine antibodies. On two-dimensional Western blots of human noncapacitated sperm extracts, the region $\sim 57 \mathrm{kDa}$ at $\mathrm{pI} \sim 6.5-8.5$, where NYD-SP28 was expected to migrate, was strongly stained by anti-NYD-SP28 serum. In capacitated sperm, these protein spots were comigrated (Fig. 7). However, these protein spots were not recognized by anti-phosphotyrosine, anti-phosphoserine and anti-phosphothreonine antibodies (data not shown). Therefore, the nature of the modification remains to be elucidated.

\section{Discussion}

In this study, a novel human gene, NYD-SP28, was found and designated and was expressed more abundantly in human adult testis than in fetal testis. Its cDNA consisted of $1798 \mathrm{bp}$ with an open reading frame of $1455 \mathrm{bp}$. Bioinformatic analysis showed that nucleotide and protein sequences of human NYD-SP28 were highly homologous with those in the mouse, rat, macaque, cow and dog. This high conservation suggests an important role of NYD-SP28 during evolution.

Hybridization of cDNA microarray indicated a 3.5-fold higher expression of NYD-SP28 in adult than in fetal testes, the latter did not produce sperm. In addition, Western blotting clearly showed that NYD-SP28-encoded protein content was high in human adult testis and ejaculated spermatozoa while undetectable in human fetal testis. In 6-month-old human fetuses, spermatogenesis does not occur, only Sertoli cells and spermatogonia in the seminiferous tubules are observed. However, seminiferous tubules of human adults contain not only Sertoli cells and spermatogonia but also various spermatogenic cells (5). Taken together, these findings suggested that NYD-SP28 was not expressed in Sertoli cells and might be correlated with the function of spermatogenic cells.

Immunohistochemical analysis was performed to determine whether NYD-SP28 expression in testis is specific to spermatogenic cells and/or somatic cells. The results showed that NYD-SP28 was exclusively expressed in the cytoplasm of spermatogenic cells rather than interstitial cells in normal human testes. Further evidence for the subcellular localization of NYD-SP28 protein came from the expression of GFP-NYD-SP28 fusion protein in 7721 cells. When GFPNYD-SP28 was transfected into 7721 cells, fluorescence for NYD-SP28 at $24 \mathrm{~h}$ after transfection was visible in the cytoplasm. Thus NYD-SP28 was a cytoplasmic protein of spermatogenic cells. Western blotting showed that NYD-SP28 was also expressed in human ejaculated spermatozoa. Immunofluorescence analysis showed that NYD-SP28 was localized in the entire human sperm tail. These results suggested that NYD-SP28 might be specifically associated with the function of sperm rather than interstitial cells in the testis.

The sperm tail is highly conserved and composed of a number of cytoskeletal elements whose proper assembly is critical for sperm motility and penetration into the egg at fertilization (9-12). Before fertilization occurs, spermatozoa must undergo capacitation, a process that acquires the ability to fuse with the oocyte. Mature spermatozoa are terminally differentiated and specialized cells. They are highly compartmentalized and transcriptionally inactive, and unable to synthesize new proteins. Therefore, it could be argued that the reliance of mature spermatozoa on posttranslational modification is a means of regulating important phenomena such as sperm capacitation (3). To date, many sperm tail proteins that can be posttranslationally modified during capacitation have been identified and these modifications can regulate subcellular localization of these proteins $(13,14)$. The present study indicated that both distribution (by indirect immunofluorescence) and protein content (by Western blotting) of NYD-SP28 were not changed in the sperm tail during capacitation (data not shown). Motif analysis revealed that NYD-SP28 protein contained two glycosylation sites, two myristoylation sites, and a cluster of phosphorylation sites, including six protein kinase $\mathrm{C}$ (PKC) phosphorylation sites, 11 casein kinase II phosphorylation sites and one tyrosine kinase phosphorylation site. We postulated that NYD-SP28 might regulate the function of sperm by posttranslational modification(s). To test this hypothesis, expression of NYDSP28 protein in noncapacitated and capacitated human spermatozoa was examined by 2-D SDS-PAGE and Western blotting. Surprisingly, a cluster of NYD-SP28 spots with the same molecular weight ( $57 \mathrm{KDa})$ were detected in noncapacitated spermatozoa. In capacitated spermatozoa, these spots were co-migrated due to molecular weight, and some acidic spots were lost in capacitated spermatozoa. Anomalous migration in SDS-PAGE due to $\mathrm{pI}$ and molecular weight has also been reported for several proteins (15-19). In addition, posttranslational modifications like phosphorylation and glycosylation are known to affect protein migration and thus, lead to anomalous estimates of the molecular weight and pI of proteins $(8,20)$. Further studies are required to characterize these posttranslational modifications. Considering the role of posttranslationally modified proteins in sperm flagella during capacitation, these results suggest an important role of NYDSP28 protein in human sperm capacitation.

In summary, the primary structure, expression, and localization of a novel human protein NYD-SP28 is reported. The findings indicate that NYD-SP28 is expressed in a development-dependent manner, localized in spermatogenic cell cytoplasm and human spermatozoa tail, and posttranslationally modified during sperm capacitation, which raises a possibility that NYD-SP28 might play an important role during capacitation and ultimately contribute to the success of fertilization. Further studies will be conducted to define the role of NYD-SP28 and explore its mechanisms in the regulation of sperm capacitation.

\section{Acknowledgements}

This work was supported by grants from the China National 973 program (2006CB504002), the Chinese National Prominent Youth Foundation (30425006) and the Special Purpose for Prophase Investigation of Great Fundamental Research (2004cca06800).

\section{References}

1. Yudin AI, Tollner TL, Li MW, Treece CA, Overstreet JW and Cherr GN: ESP13.2, a member of the beta-defensin family, is a macaque sperm surface-coating protein involved in the capacitation process. Biol Reprod 69: 1118-1128, 2003.

2. de Lamirande E, Leclerc P and Gagnon C: Capacitation as a regulatory event that primes spermatozoa for the acrosome reaction and fertilization. Mol Hum Reprod 3: 175-194, 1997. 
3. Baker MA, Witherdin R, Hetherington L, Cunningham-Smith K and Aitken RJ: Identification of post-translational modifications that occur during sperm maturation using difference in twodimensional gel electrophoresis. Proteomics 5: 1003-1012, 2005.

4. Sha JH, Zhou ZM and Li JM, et al: Identification of testis development and spermatogenesis-related genes in human and mouse testes using cDNA arrays. Mol Hum Reprod 8: 511-517, 2002.

5. Cheng LJ, Li JM, Chen J, et al: NYD-SP16, a novel gene associated with spermatogenesis of human testis. Biol Reprod 68: 190-198, 2003.

6. Zheng Y, Zhou ZM, Yin LL, Li JM and Sha JH: Molecular cloning and characterization of a novel splicing variant of PIASx. Acta Pharmacol Sin 25: 1058-1064, 2004.

7. Xu M, Xiao JH, Chen J, et al: Identification and characterization of a novel human testis-specific Golgi protein, NYD-SP12. Mol Hum Reprod 9: 9-17, 2003.

8. Wang L, Zhu YF, Guo XJ, et al: A two-dimensional electrophoresis reference map of human ovary. J Mol Med 83: 812-821, 2005.

9. Kierszenbaum AL: Sperm axoneme: a tale of tubulin posttranslation diversity. Mol Reprod Dev 62: 1-3, 2002.

10. Naz RK: Involvement of protein serine and threonine phosphorylation in human sperm capacitation. Biol Reprod 60: 1402-1409, 1999.

11. Urner F and Sakkas D: Protein phosphorylation in mammalian spermatozoa. Reproduction 125: 17-26, 2003.

12. Inaba K: Molecular architecture of the sperm flagella: molecules for motility and signaling. Zoolog Sci 20: 1043-1056, 2003.
13. Turner RM, Musse MP, Mandal A, et al: Molecular genetic analysis of two human sperm fibrous sheath proteins, AKAP4 and AKAP3, in men with dysplasia of the fibrous sheath. J Androl 22: 302-315, 2001.

14. Naaby-Hansen S, Mandal A, Wolkowicz MJ, et al: CABYR, a novel calcium-binding tyrosine phosphorylation-regulated fibrous sheath protein involved in capacitation. Dev Biol 242: 236-254, 2002 .

15. Graceffa P, Jancso A and Mabuchi K: Modification of acidic residues normalizes sodium dodecyl sulfate-polyacrylamide gel electrophoresis of caldesmon and other proteins that migrate anomalously. Arch Biochem Biophys 297: 46-51, 1992.

16. Armstrong DJ and Roman A: The anomalous electrophoretic behavior of the human papillomavirus type $16 \mathrm{E} 7$ protein is due to the high content of acidic amino acid residues. Biochem Biophys Res Commun 192: 1380-1387, 1993.

17. Chang LS, Chou L and Chang CC: Structural determinants of cobrotoxin affect its mobility on sodium dodecyl sulfate polyacrylamide gel. Biochem Mol Biol Int 40: 1227-1232, 1996.

18. Venkatesh $\mathrm{J}$ and Oommen A: A molecular weight study of adenosine deaminase. Indian J Biochem Biophys 35: 16-20, 1998.

19. Siva AB, Sundareswaran VR, Yeung CH, Cooper TG and Shivaji S: Hamster contraception associated protein 1 (CAP1). Mol Reprod Dev 68: 373-383, 2004.

20. Wistow G, Roquemore E and Kim HS: Anomalous behavior of beta B1-crystallin subunits from avian lenses. Curr Eye Res 10: 313-319, 1991. 